\title{
THE ISPRS BENCHMARK ON INDOOR MODELLING
}

\author{
K. Khoshelham ${ }^{\text {a, } *, \text { L. Díaz Vilariño }}{ }^{\text {b,c }}$, M. Peter ${ }^{\text {d }}$, Z. Kang ${ }^{\text {e }}$, D. Acharya ${ }^{\text {a }}$ \\ ${ }^{a}$ Dept. of Infrastructure Engineering, The University of Melbourne, Parkville 3010 Australia - k.khoshelham@unimelb.edu.au \\ ${ }^{\mathrm{b}}$ Applied Geotechnologies Group, Dept. of Natural Resources and Environmental Engineering, University of Vigo, Spain - \\ lucia@uvigo.es \\ ${ }^{\mathrm{c}}$ GIS Technology, OTB Research Institute for the Built Environment, Delft University of Technology, Julianalaan 134, Delft, \\ Netherlands - L.Diaz-Vilarino@tudelft.nl \\ ${ }^{\mathrm{d}}$ Dept. of Earth Observation Science, Faculty of Geo-Information Science and Earth Observation (ITC), University of Twente, The \\ Netherlands - m.s.peter@utwente.nl \\ ${ }^{\mathrm{e}}$ Dept. of Remote Sensing and Geo-Information Engineering, School of Land Science and Technology, China University of \\ Geosciences, Beijing 100083, China --zzkang@cugb.edu.cn
}

\section{Commission IV, WG IV/5}

KEY WORDS: 3D modelling, Point cloud, BIM, Quality, Accuracy, Evaluation, Performance, Automation, Indoor navigation, Geometric reconstruction, Semantics.

\begin{abstract}
:
Automated generation of 3D indoor models from point cloud data has been a topic of intensive research in recent years. While results on various datasets have been reported in literature, a comparison of the performance of different methods has not been possible due to the lack of benchmark datasets and a common evaluation framework. The ISPRS benchmark on indoor modelling aims to address this issue by providing a public benchmark dataset and an evaluation framework for performance comparison of indoor modelling methods. In this paper, we present the benchmark dataset comprising several point clouds of indoor environments captured by different sensors. We also discuss the evaluation and comparison of indoor modelling methods based on manually created reference models and appropriate quality evaluation criteria. The benchmark dataset is available for download at: http://www2.isprs.org/commissions/comm4/wg5/benchmark-on-indoor-modelling.html.
\end{abstract}

\section{INTRODUCTION}

With the growth of urban population and the prevalence of large public buildings there is an increasing demand for up-todate spatial information of indoor environments. While traditionally 2D floor plans have been regarded as the main source of indoor spatial information, advanced location-based services such as navigation assistance and emergency response require semantically rich $3 \mathrm{D}$ models of indoor environments.

The generation of 3D indoor models is a challenging task. Point clouds captured by lidar sensors and range cameras have been the main source of data for the generation of indoor models. Manual generation of indoor models from point cloud data is, however, a labour-intensive, slow and expensive process. To address this issue, a number of methods have been developed for automated generation of 3D indoor models from point clouds (Tang et al., 2010).

Although the performance of these methods has been demonstrated on various datasets in the literature, a comparative evaluation of these methods has not been possible so far. One reason for this is the lack of a freely available benchmark dataset representing indoor environments of various complexities. A second reason is the lack of a standard evaluation framework for measuring the performance of different indoor modelling methods. Thomson and Boehm (2014) provided a benchmark dataset consisting of point clouds and reference models of a corridor and an office room. While this dataset is useful for the development and testing of indoor modelling methods, it represents very simple environments, and does not include a comprehensive set of evaluation criteria to allow comparison of different indoor modelling methods.

The ISPRS benchmark on indoor modelling addresses the above issues. Led by WG IV/5, this scientific initiative aims to provide a public dataset of indoor point clouds, and organise a benchmark test for the evaluation and comparison of indoor modelling methods based on manually created reference models and appropriate quality evaluation criteria.

The paper proceeds with the description of the benchmark dataset in Section 2 and the reference models in Section 3. Section 4 presents the evaluation method. Section 5 discusses practical considerations for the implementation of the benchmark test. The paper concludes with some remarks in Section 6.

\section{DATASET}

The dataset consists of five point clouds captured by different sensors in indoor environments of various complexities. A visualization of the point clouds can be seen in Figure 1. Table 1 summarizes the specifications of the point clouds, and Table 2 presents the technical characteristics of the sensors. The general characteristics of the indoor environments represented by the point clouds are described in the following paragraphs.

\footnotetext{
* Corresponding author
} 
TUB1. This point cloud was captured in one of the buildings of the Technische Universität Braunschweig, Germany, using the Viametris iMS3D system. The data includes both the point cloud and the trajectory of the sensor during the acquisition, and both files contain timestamps. The indoor scene comprises 10 rooms on one floor, which are enclosed by walls with different thicknesses. The scene contains 23 doors, both open and closed, and 7 windows. The building is not furnished, so, the level of clutter, defined as the amount of points belonging to elements that do not constitute the building structure, is low, and it mostly corresponds to the presence of people during the survey.

TUB2. This point cloud was captured in the same building as TUB1. However, in this case, the sensor Zeb-Revo was used to perform the survey across two floors connected by a staircase. The data consist of the point cloud and the corresponding sensor trajectory, both including timestamps. The first level of the building contains 14 rooms, 8 windows and 23 doors (both open and closed, simple and double), while the second level includes 10 rooms with 13 windows and 28 doors (both open and closed, simple and double). Walls have different thicknesses and ceilings have different heights. As in the previous point cloud, the level of clutter is low.

Fire Brigade. This point cloud was captured in the office of fire brigade in Delft, The Netherlands. The data acquisition was performed using a Terrestrial Laser Scanner, Leica C10 (Sirmacek, et al, 2016). The indoor scene contains 9 rooms on the same level, 10 doors and 53 windows. The level of clutter in this point cloud is high due to the presence of furniture. The point cloud also contains gaps caused by occlusion due to the static mode of the laser scanning. A complexity of this scene is the presence of curtain walls that can challenge the reconstruction process, especially when they contain windows.

UVigo. This point cloud represents one room and an entrance hall captured at the University of Vigo, Spain. The survey was performed by a prototype of a backpack-based mobile mapping system (Filgueira et al, 2016), providing a point cloud and the trajectory of the sensor both including timestamps. The scene includes one curtain wall, 20 windows and 7 simple doors (both open and closed), of which two belong to an elevator. The scene also contains stairs to the second floor and several columns with circular cross-section in the middle. The ceiling has different heights and the level of clutter is moderate.

UoM. This point cloud was acquired by the sensor Zeb-1 in block B of the engineering building of the University of Melbourne, Australia. The indoor scene comprises 7 rooms on the same floor and 14 doors (both open and closed, single and double), with some walls having different thicknesses. Windows are not visible in this point cloud (they were covered by window blinds). The scene also contains stairs descending to a lower level. The level of clutter is moderate due to the presence of several pieces of furniture.

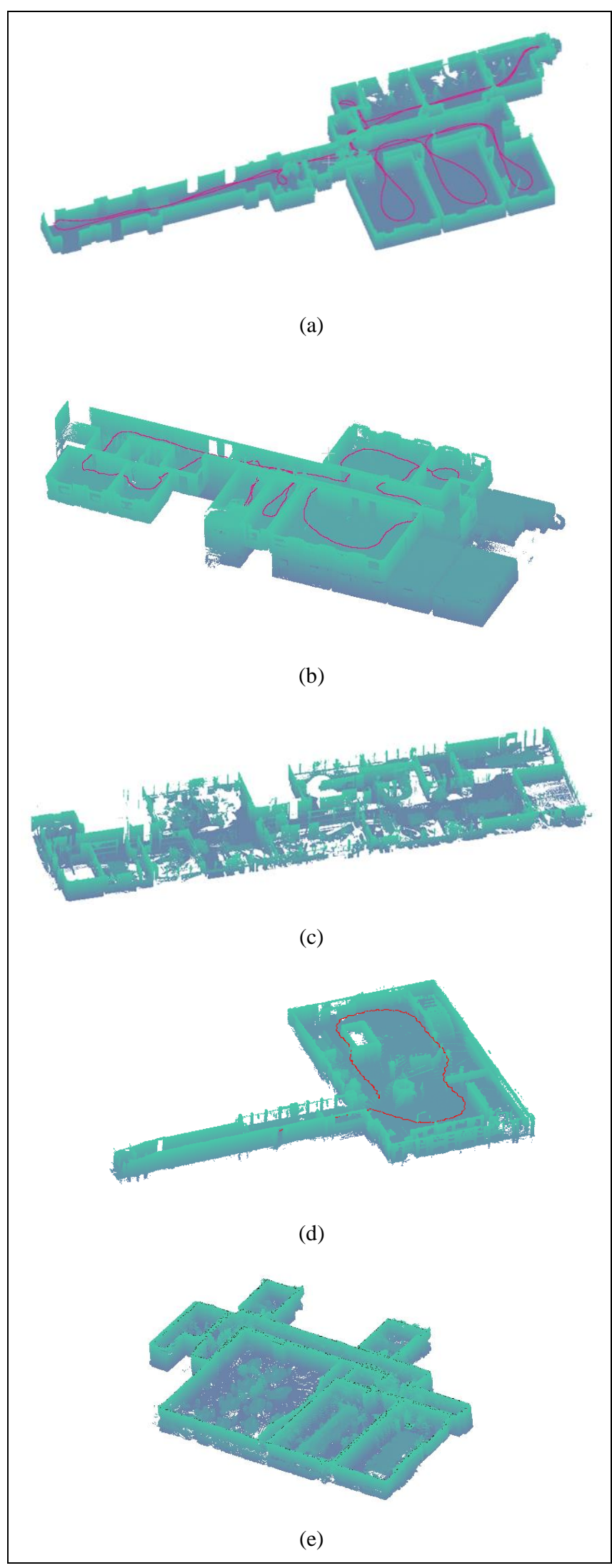

Figure 1. Screenshots of the point clouds: (a) TUB1; (b) TUB2;

(c) Fire Brigade; (d) UVigo; (e) UoM. Curves in red colour represent sensor trajectories. 


\begin{tabular}{|l|c|c|c|c|c|}
\hline \multicolumn{1}{|c|}{ Dataset } & TUB1 & TUB2 & $\begin{array}{c}\text { Fire } \\
\text { Brigade }\end{array}$ & UVigo & UoM \\
\hline Sensor & $\begin{array}{c}\text { Viametris } \\
\text { iMS3D }\end{array}$ & $\begin{array}{c}\text { ZEB } \\
\text { REVO }\end{array}$ & $\begin{array}{c}\text { TLS Leica } \\
\text { C10 }\end{array}$ & $\begin{array}{c}\text { UVigo } \\
\text { Backpack }\end{array}$ & ZEB1 \\
$\begin{array}{l}\text { Number of } \\
\text { points }\end{array}$ & $33.6 \times 10^{6}$ & $21.6 \times 10^{6}$ & $14.1 \times 10^{6}$ & $14.9 \times 10^{6}$ & $13.9 \times 10^{6}$ \\
$\begin{array}{l}\text { Mean point } \\
\text { spacing (m) }\end{array}$ & 0.005 & 0.008 & 0.011 & 0.010 & 0.007 \\
$\begin{array}{l}\text { Colour } \\
\text { Trajectory }\end{array}$ & No & No & Yes & No & No \\
Clutter & Low & Low & No & Yes & No \\
High & Moderate & Moderate \\
\hline
\end{tabular}

Table 1. Specifications of the point clouds

\begin{tabular}{|l|c|c|c|c|c|}
\hline Sensor & $\begin{array}{l}\text { Viametris } \\
\text { iMS3D }\end{array}$ & $\begin{array}{c}\text { ZEB } \\
\text { REVO }\end{array}$ & $\begin{array}{c}\text { TLS Leica } \\
\text { C10 }\end{array}$ & $\begin{array}{c}\text { UVigo } \\
\text { Backpack }\end{array}$ & ZEB1 \\
\hline $\begin{array}{l}\text { Max range } \\
\text { Speed } \\
\text { (points/sec) } \\
\text { Horizontal } \\
\text { Angular } \\
\text { Resolution (deg) }\end{array}$ & $80 \mathrm{~m}$ & $30 \mathrm{~m}$ & $300 \mathrm{~m}$ & $100 \mathrm{~m}$ & $30 \mathrm{~m}$ \\
$\begin{array}{l}\text { Vertical } \\
\text { Angular } \\
\text { Resolution (deg) } \\
\text { Angular FOV } \\
\text { (deg) }\end{array}$ & 0.25 & 0.625 & 0.01 & $0.1-0.4$ & 0.25 \\
$\begin{array}{l}\text { Relative } \\
\text { Accuracy } \\
\text { Absolute } \\
\text { Accuracy }\end{array}$ & $360 \times 360$ & $270 \times 360$ & $270 \times 360$ & $30 \times 360$ & $270 \times 150$ \\
\hline
\end{tabular}

Table 2. Technical specifications of the sensors

\section{REFERENCE MODELS}

The reference models were created manually in Autodesk Revit $^{\mathrm{TM}}$ software. Before importing to Revit ${ }^{\mathrm{TM}}$, the point clouds were pre-processed in CloudCompare ${ }^{\mathrm{TM}}$ software. The preprocessing involves cleaning the point cloud (removing noise and reflection points), and rotating it such that the walls, floors and ceilings are aligned with the $\mathrm{X}, \mathrm{Y}, \mathrm{Z}$ axes of the point cloud.

The pre-processed point cloud is then imported to Autodesk Revit $^{\mathrm{TM}}$ software for the geometric reconstruction of building elements. The geometric reconstruction consists of two main steps. The first step involves the placement of the levels. Each level of the building is identified by making vertical sections of the point cloud and inspecting the density of the points. For each level, multiple horizontal sections are made to facilitate the placement of building elements.

The second step is the placement of building elements into each level. Guided by the horizontal sections, walls are first placed into the model. Afterwards, stairs, floors, windows, columns, doors and ceilings are added. The height of each element is determined by inspecting several vertical sections of the point cloud. Raised floors and false ceilings are identified in the vertical sections, and are modelled by multi-height elements. Parallelism and orthogonality of the walls are maintained during the geometric reconstruction. Figure 2 shows a horizontal section of the point cloud used for the placement of walls, and a vertical section used for the placement of floors, ceilings, doors and windows.
During the manual reconstruction, floor plans were used to gain a better understanding of the environment. However, no measurements from the floor plans were used during the modelling procedure. Moreover, only building elements that were visible in the point cloud were reconstructed and no supplementary information from the floor plans was used to reconstruct building elements that were not captured in the point cloud. The final reconstructed models contain walls, floors, ceilings, stairs, windows, doors, columns and railings. All other objects were ignored. Figure 3 shows the final reference models.

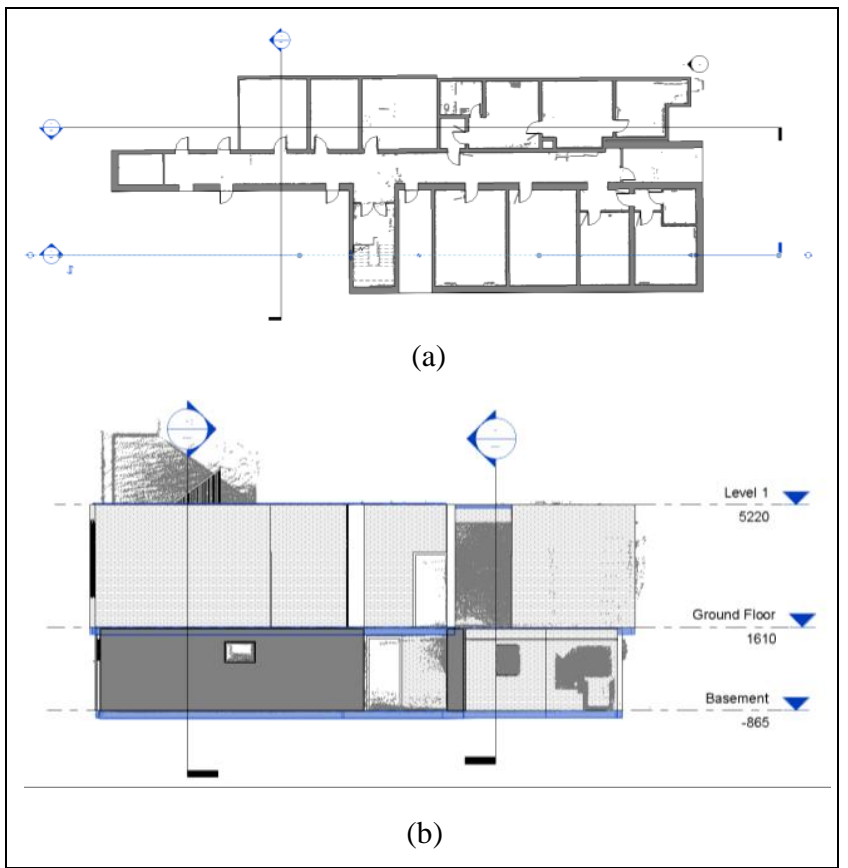

Figure 2. Manual reconstruction of building elements from a point cloud: (a) a horizontal section for the placement of walls, doors and stairs; (b) a vertical section for the placement of floors, ceilings, doors and windows.

\section{EVALUATION CRITERIA}

Apart from factors such as computational efficiency and level of automation, the performance of an indoor modelling algorithm can be evaluated on the basis of the quality of its reconstructed models. In principle, an indoor model consists of three constituent components:

- Geometric elements (e.g., walls, floors and ceilings);

- Semantics (e.g., type, function, or material of the elements);

- Spaces and topological relations between the spaces (e.g., adjacency and connectivity of the rooms).

In practice, most existing methods reconstruct geometric elements only (Becker et al., 2015; Díaz-Vilariño et al., 2015; Mura et al., 2016; Oesau et al., 2014; Sanchez and Zakhor, 2012; Thomson and Boehm, 2015; Valero et al., 2012; Xiao and Furukawa, 2012; Xiong et al., 2013), while a few can model spaces and the topological relations between them (Khoshelham and Díaz-Vilariño, 2014; Tran et al., 2017). Nonetheless, a comprehensive evaluation should take into account all three components of an indoor model. 


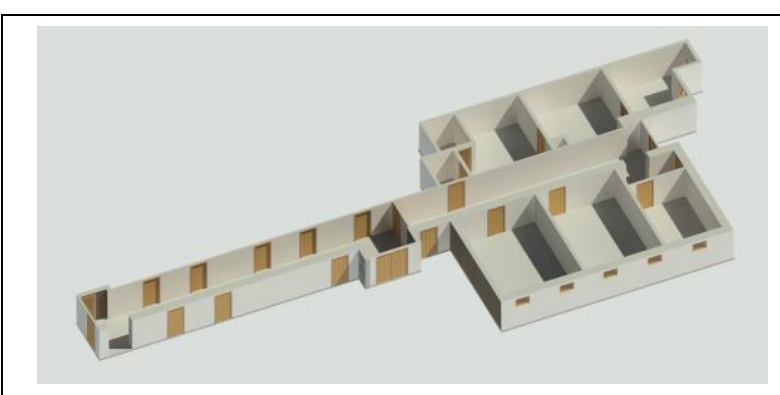

(a)

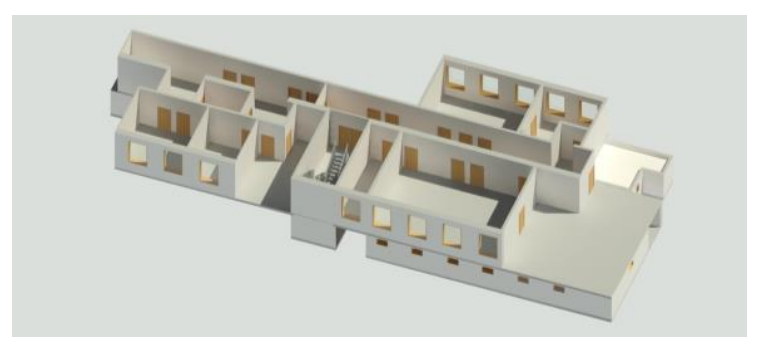

(b)

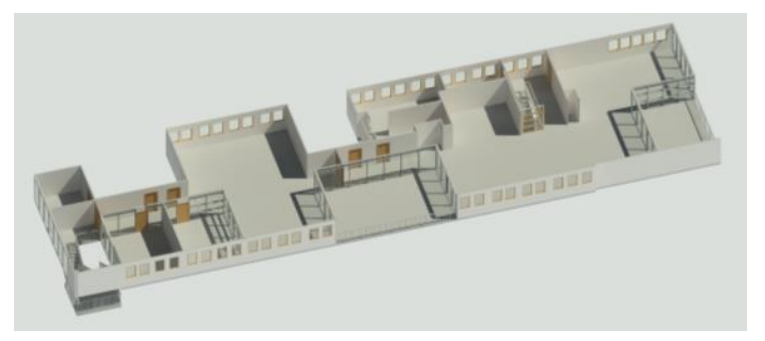

(c)

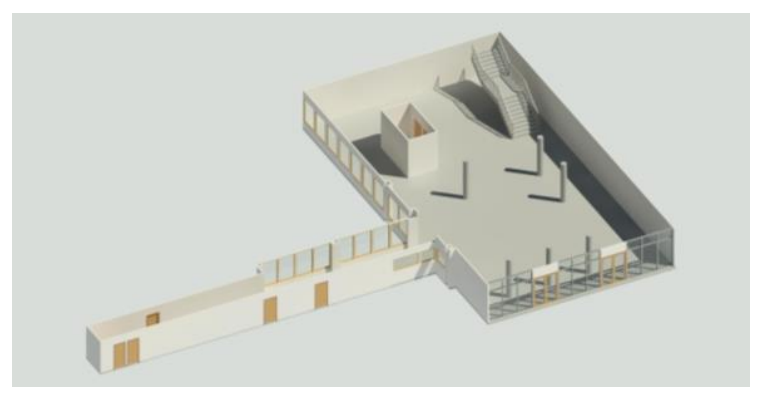

(d)

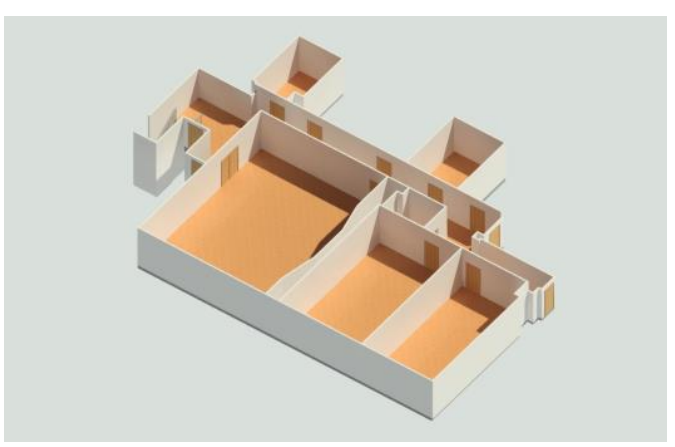

(e)

Figure 3. Perspective views of the reference models: (a) TUB1; (b) TUB2; (c) Fire Brigade; (d) UVigo; (e) UoM. Ceilings have been removed for better visualization of the interior.
We propose a framework consisting of quantitative and qualitative criteria for the evaluation of geometric elements as well as semantics and topological relations in an indoor model. The semantics and topological relations are evaluated qualitatively by a panel of experts, who will inspect the models and check the presence and correctness of the semantic attributes as well as the spaces and their topological relations. The geometric elements are evaluated quantitatively through a comparison between the reference model and the automatically reconstructed model, hereafter referred to as the source model.

The quantitative evaluation is based on three criteria: completeness, correctness and accuracy of the reconstructed elements. Completeness describes the extent to which the geometric elements of the reference model are present in the source model. Let $R$ and $S$ denote the set of elements in the reference and the source model respectively. Completeness is then defined as:

$$
\text { Completeness }=\frac{|R \cap S|}{|R|}
$$

where |.| denotes the number of elements in a set.

Correctness describes the extent to which the geometric elements of the source model are present in the reference model:

$$
\text { Correctness }=\frac{|R \cap S|}{|S|}
$$

Accuracy measures the closeness of the reconstructed elements in the source model to their corresponding elements in the reference model. It is typically measured by comparing the parameters of the corresponding elements in the source and reference models. This approach is suitable for models that contain parametric solids, e.g., those compliant with the IFC standard. However, we recognise that some methods produce surface models, which contain surfaces (rectangular patches or triangular meshes) rather than parametric solids. To be able to measure the accuracy of both parametric and non-parametric models, we define the accuracy based on the distances between the vertices in the source model and their corresponding planar faces in the reference model. We use the signed perpendicular point-plane distance defined as (Khoshelham, 2016):

$$
v_{i j}=\boldsymbol{\pi}_{j}^{T} \tilde{\mathbf{p}}_{i}
$$

where $\tilde{\mathbf{p}}_{i}$ and $\boldsymbol{\pi}_{j}$ denote the homogeneous representation of the vertex point $i$ and the plane $j$ respectively. The accuracy is then defined as the median of absolute distances:

$$
\text { Accuracy }=\operatorname{Med}\left(v_{i j}\right)
$$

\section{PRACTICAL CONSIDERATIONS}

As part of the ISPRS benchmark on indoor modelling, we organise a test to evaluate and compare the performance of indoor modelling methods. The benchmark dataset is available for download from the ISPRS website: http://www2.isprs.org/commissions/comm4/wg5/benchmarkon-indoor-modelling.html. 
Interested participants are invited to use the benchmark dataset to test their methods, and submit their reconstructed indoor models for evaluation. The evaluation results will be published on the ISPRS website.

To facilitate the quantitative evaluation, we encourage the participants to use the IFC standard and file format (Liebich, 2009; Ward et al., 2012). This would require reconstructing indoor models as a set of parametric solids. Surface models are also accepted; however, for the quantitative evaluation surfaces will be treated as solids with zero thickness.

For a fair quantitative evaluation, it is necessary to specify which geometric elements will be included in the evaluation. In this benchmark test, we limit the quantitative evaluation to the following basic elements that are present in most indoor environment: walls, floors, ceilings, doors and windows. Other elements, such as stairs and columns, are excluded from the quantitative evaluation.

It is also important to have a clear definition for each building element. According to Oxford dictionary, a room is defined as $a$ part or division of a building enclosed by walls, floor, and ceiling. A possible ambiguity may arise from the presence of curtain walls used for enclosing spaces in some indoor environments. This occurs, for instance, in the Fire Brigade and UVigo datasets, where some curtain walls also have windows. To disambiguate, we treat curtain walls as normal walls, but exclude windows of curtain walls from the evaluation. Another possible ambiguity might arise in discerning an open door from an opening in a wall. To distinguish between the two, we consider that a door is typically lower than the height of the wall it is contained in, whereas an opening extends the entire height of the wall and reaches the ceiling. Figure 4 illustrates an example from dataset TUB1. An opening is not considered as a building element. Consequently, it does not constitute the subdivision of a space. Therefore, the small space adjacent to the opening in Figure 4 is considered an extension of the corridor.

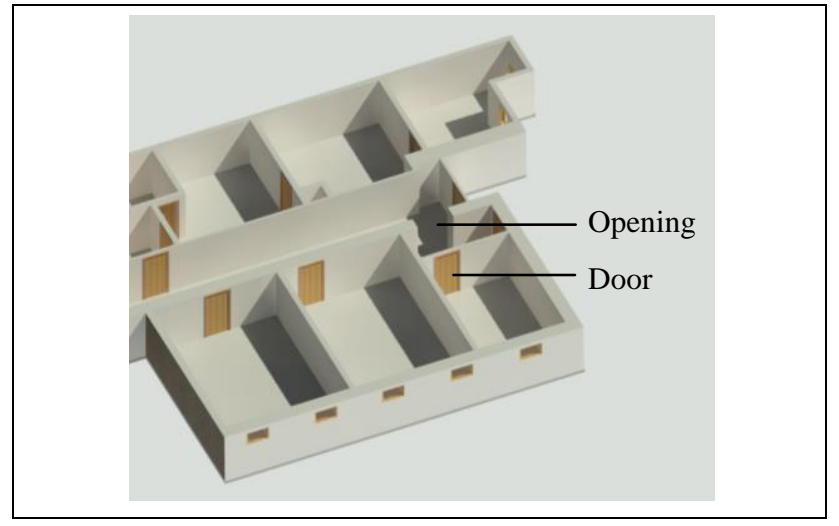

Figure 4. An example of a door and an opening in the wall.

\section{CONCLUSIONS}

This paper introduced the ISPRS benchmark on indoor modelling. We presented the benchmark dataset comprising five point clouds acquired by different sensors in indoor environments of various complexities and containing different levels of clutter. We also described a framework for the evaluation and comparison of indoor modelling methods based on manually created reference models and appropriate quality evaluation criteria. We invite all researchers involved in indoor modelling research to use the benchmark dataset to test their methods, and submit their reconstructed models for evaluation. The results of the evaluation will be published on the ISPRS website.

\section{ACKNOWLEDGEMENTS}

This work is supported by the ISPRS Scientific Initiatives 2017. The TUB1 and TUB2 datasets were provided by Markus Gerke from the Technical University of Braunschweig (Germany), with the collaboration of Viametris and Laserscanning Europe. The Fire Brigade dataset was supplied by Sisi Zlatanova from the Delft University of Technology (The Netherlands), and was acquired for the project SIMs3D funded by the Netherlands Technology Foundation (STW). The UVigo data set was provided by Pedro Arias from University of Vigo (Spain), and was acquired for the project ENGINENCY funded by the program H2020-FTIPilot-2015-1. The UoM dataset was provided by Ebadat Ghanbari Parmehr from the RMIT University (Australia). The support from Xunta de Galicia through the human resources grant ED481B 2016/079-0 is also gratefully acknowledged.

\section{REFERENCES}

Becker, S., Peter, M., Fritsch, D., 2015. Grammar-Supported 3D Indoor Reconstruction From Point Clouds for "As-Built" BIM. ISPRS Ann. Photogramm. Remote Sens. Spatial Inf. Sci. II-3/W4, 17-24.

Díaz-Vilariño, L., Khoshelham, K., Martínez-Sánchez, J., Arias, P., 2015. 3D Modeling of Building Indoor Spaces and Closed Doors from Imagery and Point Clouds. Sensors 15, 3491-3512.

Filgueira, A., Laguela, S., Arias, P., Bueno, M., 2016. Novel inspection system, backpack-based, for 3D modelling of indoor scenes. International Conference on Indoor Positioning and Indoor Navigation (IPIN). 4-7 October, 2016 (Madrid)

Khoshelham, K., Díaz-Vilariño, L., 2014. 3D modeling of interior spaces: learning the language of indoor architecture. Int. Arch. Photogramm. Remote Sens. Spatial Inf. Sci., XL-5, 321326.

Khoshelham, K., 2016. Closed-form solutions for estimating a rigid motion from plane correspondences extracted from point clouds. ISPRS Journal of Photogrammetry and Remote Sensing 114, 78-91.

Liebich, T., 2009. IFC 2x Edition 3 Model Implementation Guide. Version 2.0. High Wycombe, UK, AEC3 Ltd.

Mura, C., Mattausch, O., Pajarola, R., 2016. Piecewise-planar Reconstruction of Multi-room Interiors with Arbitrary Wall Arrangements. Computer Graphics Forum 35, 179-188.

Oesau, S., Lafarge, F., Alliez, P., 2014. Indoor scene reconstruction using feature sensitive primitive extraction and graph-cut. ISPRS Journal of Photogrammetry and Remote Sensing 90, 68-82.

Sanchez, V., Zakhor, A., 2012. Planar 3D modeling of building interiors from point cloud data, 19th IEEE International 
Conference on Image Processing (ICIP), Orlando, FL, pp. $1777-1780$

Sirmacek, B., Shen, Y., Lindenbergh, R., Zlatanova, S., Diakite, A., 2016. Comparison of Zeb1 and Leica C10 Indoor Laser Scanning Point Clouds. Int. Ann. Photogramm. Remote Sens. Spatial Inf. Sci. III-1, 143-149.

Tang, P., Huber, D., Akinci, B., Lipman, R., Lytle, A., 2010. Automatic reconstruction of as-built building information models from laser-scanned point clouds: A review of related techniques. Automation in Construction 19, 829-843.

Thomson, C., Boehm, J., 2014. Indoor Modelling Benchmark for 3D Geometry Extraction. Int. Arch. Photogramm. Remote Sens. Spatial Inf. Sci. XL-5, 581-587.

Thomson, C., Boehm, J., 2015. Automatic Geometry Generation from Point Clouds for BIM. Remote Sensing 7, 11753.

Tran, H., Khoshelham, K., Kealy, A., Díaz-Vilariño, L., 2017. Extracting Topological Relations Between Indoor Spaces From Point Clouds, ISPRS Workshop Indoor 3D 2017, Wuhan, China.
Valero, E., Adán, A., Cerrada, C., 2012. Automatic Method for Building Indoor Boundary Models from Dense Point Clouds Collected by Laser Scanners. Sensors 12, 16099-16115.

Ward, A., Benghi, C., Ee, S. and Lockley, S., 2012. The eXtensible Building Information Modelling (xBIM) Toolkit. CodePlex, Northumbria University. Available online: http://nrl.northumbria.ac.uk/13123/ (accessed on 7 July 2017).

Xiao, J., Furukawa, Y., 2012. Reconstructing the world's museums, Proceedings of the 12th European conference on Computer Vision - Volume Part I. Springer-Verlag, Florence, Italy, pp. 668-681.

Xiong, X., Adan, A., Akinci, B., Huber, D., 2013. Automatic creation of semantically rich $3 \mathrm{D}$ building models from laser scanner data. Automation in Construction 31, 325-337. 